INVESTIGACIÓN

https://doi.org/10.15198/seeci.2019.50.169-192

Recibido: 12/09/2019 --- Aceptado: 21/10/2019 --- Publicado: 15/11/2019

\title{
APLICACIONES DIGITALES PARA LA INCLUSIÓN. EL PROYECTO EUROPEO DEPIT
}

\section{DIGITAL APPLICATIONS FOR INCLUSION. THE EUROPEAN PROJECT DEPIT}

\author{
(i) (8) $\mathrm{R}^{9}$ I P Pilar Colás-Bravo: Universidad de Sevilla. España. \\ pcolas@us.es
}

(i) (8) 18 Pier Giuseppe Rossi. Universidad de Macerata. Italia. pg.rossi@unimc.it

(i) (8) $\mathrm{R}^{\mathrm{C}}$ : $1 \mathrm{P}$ Juan de Pablos-Pons. Universidad de Sevilla. España. jpablos@us.es

(D) (8) 18 Jesús Conde-Jiménez. Universidad Loyola Andalucía. España. jconde@uloyola.es

D (8) $\mathbb{R}^{R}$ P Patricia Villaciervos Moreno. Universidad de Sevilla. España. pvillaciervo@us.es

DEPIT (Designing for Personalization and Inclusion with Technologies) forma parte de un proyecto cofinanciado por el programa Erasmust de la Unión Europea, en el que participan diferentes universidades europeas. Web del proyecto: http://depit.eu

\section{RESUMEN}

En esta aportación se plantea la relevancia de la personalización y la inclusión como aspectos clave de la planificación de la enseñanza y se incide en la idoneidad de las tecnologías para apoyar esta perspectiva. Se presenta y evalúa desde la perspectiva del profesorado, el potencial de la herramienta DEPIT (Designing for Personalization and Inclusion with Technologies) cuya finalidad es apoyar la planificación y programación docente, atendiendo a la inclusión y personalización. Se recoge información del profesorado sobre su actitud hacia DEPIT, así como sobre su utilidad en la praxis docente. El valor aplicado de DEPIT se recaba a través de un foro de discusión. El análisis cuantitativo de los datos llevado a cabo mediante el programa estadístico SPSS v.24, identifica actitudes positivas del profesorado en relación al uso

\footnotetext{
${ }^{1}$ Pilar Colás-Bravo: Catedrática de Métodos de Investigación y Diagnóstico en Educación. Imparte docencia en la Universidad de Sevilla (España) en titulaciones de Grado, Masters y Doctorados. Ha dirigido más de una veintena de Tesis Doctorales y coordinado múltiples Proyectos de Investigación e Innovación. Colabora en calidad de Experta en diferentes agencias internacionales. pcolas@us.es
} 


\section{Colás-Bravo, P.; Rossi, P. G.; de Pablos-Pons, J.; Conde-Jiménez, J. y Villaciervos Moreno, P. Aplicaciones digitales para la inclusión. \\ El Proyecto Europeo DEPIT}

de herramientas digitales para elaborar la programación docente. El análisis cualitativo, realizado mediante el programa Atlas.Ti V.8, registra aspectos que los docentes valoran de la aplicación DEPIT, entre otros: "Adaptación y versatilidad a contextos educativos diversos" "Facilita la interacción y la comunicación" y "Potencia la libertad, independencia y autonomía". De los resultados obtenidos se concluye que el profesorado asume una actitud proactiva y positiva hacia la incorporación de herramientas tecnológicas para su docencia y ponen en valor el potencial de DEPIT para la programación docente.

PALABRAS CLAVE: profesorado - TIC - programación - inclusión - personalización - enseñanza y aprendizaje.

\section{ABSTRACT}

This article discusses the relevance of personalization and inclusion as key aspects of teaching planning and emphasizes the suitability of technologies to support this perspective. An app, called DEPIT (Designing for Personalization and Inclusion with Technologies), whose purpose is to support teacher planning and programming, is presented and evaluated from the perspective of the teaching staff. The information is collected from teachers regarding their attitude towards the DEPIT app, as well as its usefulness in teaching practice. The applied value of the app is collected through a discussion forum. The quantitative analysis of the data carried out by means of the statistical program SPSS v.24, identifies positive attitudes of the teaching staff in relation to the use of digital tools to elaborate the teaching programming. The qualitative analysis, carried out through the software Atlas.ti v.8, records aspects that teachers value in the application of DEPIT, among others: "Its adaptation and versatility to diverse educational contexts", "It facilitates interaction and communication" and "It promotes freedom, independence and autonomy". The results allow us to conclude that teachers assume a proactive and positive attitude towards the incorporation of technological tools for their teaching and put in value the potential of the DEPIT App for teacher programming.

KEY WORDS: teachers - ICT - programming - inclusion - personalization teaching and learning.

\section{APLICAÇÕES DIGITAIS PARA A INCLUSÃO. O PROJETO EUROPEU DEPIT}

\section{RESUMO}

Nesta aportação se propõe a relevância da personalização e à inclusão como aspectos chave da planificação do ensino e se incide na idoneidade das tecnologias para apoiar esta perspectiva. Se apresenta e avalia desde a perspectiva do professorado, 0 potencial da ferramenta DEPIT (Designing for Personalization and Inclusion with Technologies) cuja finalidade e apoiar a planificação e programação docente, atendendo a inclusão e personalização. Colhendo informação do professorado sobre sua atitude com DEPIT, assim como sobre sua utilidade na práxis docente. $O$ valor aplicado do DEPIT se consegue através de um fórum de discussão. $A$ analises 


\section{Colás-Bravo, P.; Rossi, P. G.; de Pablos-Pons, J.; Conde-Jiménez, J. y Villaciervos Moreno, P. Aplicaciones digitales para la inclusión. \\ El Proyecto Europeo DEPIT}

quantitativa dos dados estudados mediante o programa estatístico SPSS v.24, identifica atitudes positivas do professorado em relação ao uso de ferramentas digitais para elaborara a programação docente. A analises qualitativa, realizada mediante 0 programa Atlas.Ti V.8, registra aspectos que os docentes avaliam da aplicação DEPIT, entre outros: "adaptação e versatilidade a contextos educativos diversos" "Facilita a interação e a comunicação" e "potência a liberdade, independência e autonomia". Dos resultados obtidos se conclui que o professorado assume uma atitude proativa e positiva para a incorporação de ferramentas tecnológicas para sua docência e põe em valor o potencial do DEPIT para programação docente.

PALAVRAS CHAVE: professorado - TIC - programação - inclusão - personalização, ensino e aprendizagem.

\section{Cómo citar el artículo:}

Colás-Bravo, P.; Rossi, P. G.; de Pablos-Pons, J.; Conde-Jiménez, J. y Villaciervos Moreno, P. (2019). Aplicaciones digitales para la inclusión. El Proyecto Europeo DEPIT. [Digital applications for inclusion. The European Project DEPIT]. Revista de Comunicación de la SEECI, (50), 169-192. doi: http://doi.org/10.15198/seeci.2019.50.169-192

Recuperado de http://www.seeci.net/revista/index.php/seeci/article/view/618

\section{INTRODUCCIÓN}

La sociedad actual vista como una realidad globalizada y líquida, según la describe Bauman (2018), se caracteriza por su complejidad y diversidad. En ese contexto, los sistemas educativos están obligados a aportar respuestas adaptadas a esa realidad heterogénea, donde la interculturalidad es hoy un componente relevante. Como consecuencia, los conceptos de personalización e inclusión se convierten en referentes principales para las instituciones educativas y por tanto para la labor de planificación de la docencia. Este horizonte genera permanentemente nuevas exigencias educativas a las que el profesorado de todos los niveles educativos debe dar respuesta mediante un trabajo de constante actualización y el consecuente desarrollo de competencias apropiadas.

Cuando hablamos de aprendizaje personalizado nos referimos a aquellas estrategias, soluciones e intervenciones de enseñanza que se ajustan a las necesidades de cada alumno y alumna, teniendo en cuenta cómo aprenden, sus aspiraciones, capacidades y percepciones, así como sus diferencias culturales, intereses y conocimientos previos (Bosada, 2018). Hablamos consecuentemente que debe partirse de un modelo pedagógico centrado en el alumnado, el cual ha cobrado fuerza en los últimos años, especialmente con el apoyo de las tecnologías de información y comunicación (TIC), que han permitido personalizar la enseñanza de manera más atractiva, motivadora y eficaz para que los estudiantes se conviertan en protagonistas de su propio aprendizaje. Las tecnologías facilitan la creación de itinerarios personalizados de los estudiantes con base a perfiles y necesidades diferentes proporcionando así un entorno personal flexible mediante el cual cada uno puede avanzar a su ritmo adquiriendo un papel activo en su propia educación. 


\section{Colás-Bravo, P.; Rossi, P. G.; de Pablos-Pons, J.; Conde-Jiménez, J. y Villaciervos Moreno, P. Aplicaciones digitales para la inclusión. \\ El Proyecto Europeo DEPIT}

\section{Por otra parte,}

la inclusión educativa es un proceso orientado a garantizar el derecho a una educación de calidad a todos los estudiantes en igualdad de condiciones, prestando especial atención a quienes están en situación de mayor exclusión o en riesgo de ser marginados. El desarrollo de escuelas inclusivas, que acojan a todos los estudiantes, sin ningún tipo de discriminación, y favorezcan su plena participación, desarrollo y aprendizaje, es una poderosa herramienta para mejorar la calidad de la educación y avanzar hacia sociedades más justas y cohesionadas" (Organización de Estados Iberoamericanos para la Educación, la Ciencia y la Cultura (OEI), s.f.).

La cultura inclusiva dirigida a garantizar la igualdad de oportunidades para todos en materia de educación, se convierte en el objetivo principal a alcanzar en las aulas y en un desafío a escala mundial. En este sentido, la UNESCO en el Marco de Acción para la realización del Objetivo de Desarrollo Sostenible 4 de la Agenda 2030 en lo relativo a la Educación, hace hincapié en que la inclusión y la igualdad son los cimientos de una enseñanza de calidad destacando la importancia de "Garantizar una educación inclusiva y equitativa de calidad y promover oportunidades de aprendizaje permanente para todos".

La presencia de las tecnologías en la escuela facilita este cometido permitiéndonos desarrollar nuevas estrategias de aprendizaje que contribuyan directamente al desarrollo de aulas inclusivas. Las Tecnologías de la Información y Comunicación (TIC) se consideran herramientas de aprendizaje de naturaleza flexible y altamente motivadoras $y$, por tanto, de las que pueden beneficiarse un gran número de alumnos con diferentes niveles cognitivos, capacidades de aprendizaje y origen (Siu, y Lam, 2012; Grande y González, 2015). En este sentido, integrar metodologías innovadoras y herramientas digitales, puede ofrecernos un amplio abanico de posibilidades para reorientar las prácticas educativas tradicionales pensando en construir entornos cooperativos de educación inclusiva.

Las dotaciones tecnológicas que se están haciendo a nivel institucional pretenden que la escuela incorpore los avances de la Sociedad de la Información. Las herramientas digitales y los materiales interactivos constituyen una fuente de recursos didácticos que dotan de un componente motivacional añadido a las actividades escolares, favoreciendo la atención a la diversidad y la creación de ambientes de aprendizaje dinámicos y flexibles. En esta línea programas europeos de investigación apuestan por apoyar proyectos destinados a diseñar, experimentar y evaluar aplicaciones digitales que apoyen al profesorado en sus funciones y faciliten una educación de calidad, basada en la inclusión y la sostenibilidad.

\subsection{La importancia de programar la enseñanza}

La preparación de un proyecto educativo, implícito o explícito, siempre ha caracterizado la acción de los docentes también porque identifica cada acción humana y deriva de la necesidad de anticipación (Berthoz, 2011) y previsión. Ya 


\section{Colás-Bravo, P.; Rossi, P. G.; de Pablos-Pons, J.; Conde-Jiménez, J. y Villaciervos Moreno, P. Aplicaciones digitales para la inclusión. \\ El Proyecto Europeo DEPIT}

Dewey declaró que carencias en esta vertiente muy a menudo son el origen del fracaso de las prácticas educativas:

A la falta de una planificación suficientemente reflexiva de antemano. Las causas de tal falta son variadas. La única, que es particularmente importante mencionar a este respecto, es la idea de que tal planificación anticipada es innecesaria e incluso que es intrínsecamente hostil a la libertad legítima de los instruidos. (...) Por el contrario, incumbe al educador el deber de instituir: mucho más inteligente $y$, en consecuencia, más difícil, la tarea de planificación. Debe examinar las capacidades y necesidades del conjunto particular de individuos con los que está tratando y, al mismo tiempo, debe organizar las condiciones que proporcionan el tema o el contenido para las experiencias que satisfacen estas necesidades y desarrollar estas capacidades. La planificación debe ser lo suficientemente flexible como para permitir el juego libre para la individualidad de la experiencia $y$, sin embargo, lo suficientemente firme como para dar una dirección hacia el desarrollo continuo del saber. (Dewey, 1938, p. 46).

Hoy, casi un siglo después de las palabras de Dewey, el diseño requiere más atención debido a la complejidad del aula donde se cruzan diferentes culturas, habilidades y conocimientos. Esta complejidad requiere, en particular, una planificación explícita compartida tanto con la comunidad docente como con los estudiantes. En este sentido, hablamos cada vez más de la planificación conjunta.

\subsection{Uso de tecnologías digitales para programar una enseñanza inclusiva}

Actualmente la tecnología es una llave para crear espacios donde estudiantes y docentes comparten su día a día. Los medios tecnológicos y virtuales son propuestos para el docente como andamios en el proceso de enseñanza-aprendizaje, y comienza a ser algo más que la herramienta o el medio (Gámiz y Gallego, 2016).

Las TIC apoyan al profesorado en su docencia desde diversas perspectivas, una de ellas es permitir y facilitar el diseño y planificación de su trabajo (Onrubia, 2016). La inclusión educativa es ya una realidad en el sistema educativo actual. Además, ahora comienza a tomar forma y protagonismo el concepto de personalización del aprendizaje, donde el logro de cada estudiante depende de si el modelo de enseñanza-aprendizaje usado es motivador y responde a las expectativas y necesidades formativas de los educandos. Elementos tecnológicos útiles para este propósito son las plataformas virtuales, Apps interactivas, foros, debates, o actividades participativas y colaborativas en un marco pedagógico diseñado a priori por el propio profesorado. Estas herramientas de gestión y planificación mejoran las posibilidades por las que optar (De Pablos, Colás, López y García, 2019).

Para ejecutar la personalización, según Touron (2019), el docente puede servirse de la ayuda de la tecnología. Una de las principales razones que explica el interés en los últimos años por la personalización es el fuerte desarrollo de las tecnologías de la información. Las TIC ayudan actualmente al sistema educativo a dar un rol participativo y activo tanto a docente como a alumnado. Por tanto, el uso de nuevas 


\section{Colás-Bravo, P.; Rossi, P. G.; de Pablos-Pons, J.; Conde-Jiménez, J. y Villaciervos Moreno, P. Aplicaciones digitales para la inclusión. \\ El Proyecto Europeo DEPIT}

tecnologías proporciona un paso más allá para generar cambio en materia educativa. Este cambio es evidente en la personalización y adaptación del proceso de enseñanzaaprendizaje tanto al docente como a sus estudiantes. Los defensores de la personalización a través de la tecnología sostienen principalmente dos argumentos: la importancia de contemplar el itinerario y el ritmo. Si se respeta y se da la posibilidad al estudiante de controlar lo que aprende y a qué velocidad y en qué momento aprende, el aprendizaje será mayor y de mejor calidad. Para muchos la personalización, ya sea de un diseño de aprendizaje o de un contenido a aprender, está actualmente vinculada a los dispositivos tecnológicos y a los datos o información. Su principal fortaleza estriba en la capacidad de disponer de múltiples recursos e informaciones, lo que permite mejorar el proceso de aprendizaje individual y también el colectivo.

De forma que el rol de las TIC en este contexto se centra en la creación, distribución y adaptación de recursos flexibles al usuario, en este caso estudiante y docente, proveyendo de mecanismos al último para resolver problemáticas y cuestiones que en el día a día escolar se presentan. Esto último incorpora acceso personalizado al contenido educativo, adaptándose a ritmos, estilos y necesidades de aprendizaje. De esta forma, el estudiante puede acceder a diversidad de recursos educativos a su vez que encuentra un refuerzo y apoyo en los casos de bajo rendimiento o de un nivel más lento de aprendizaje, así como ampliar conocimientos sin limitaciones a aquellos que puedan y quieran ir más allá (Fernández, 2017).

Diseñar en términos de personalización del aprendizaje es un mecanismo de construcción de contenidos y competencias mediados por nuevas tecnologías que también incluye la generación de situaciones educativas específicas. Este modelo que lleva al profesorado a crear itinerarios curriculares y formativos adaptados a su nivel competencial, a su grupo-clase, a los subgrupos que existen en ella, o incluso a cada uno de los estudiantes que la conforman, es una estrategia necesaria para el sistema educativo mundial (Pineda y Castañeda, 2013). Por tanto, es clave entender las tecnologías como herramientas y al mismo tiempo mecanismos que ayudan a crear espacios, recursos y procesos personalizados potenciando la relación entre profesorado y estudiantes (Cubeles y Riu, 2018).

Uno de los medios tecnológicos que más versatilidad admite en estos términos es la plataforma virtual o espacio virtual compartido entre alumnado-docente (Caminal y Puigcerver, 2017; De Pablos, et al., 2019). Por otra parte, las Apps también aportan diversificación de estímulos y recursos que incentivan el desarrollo de las competencias de los estudiantes con distintos ritmos de aprendizaje (Caminal y Puigcerver, 2017; Molano, Sánchez y Castillo, 2012).

Pero todos estos recursos deben ser seleccionados, organizados y estructurados por el docente para alcanzar los objetivos formativos. Esta tarea toma forma y se visualiza a través de la programación. Por tanto, la programación es el primer eslabón del proceso educativo que conlleva y refleja el modelo pedagógico que guía al profesorado. En este sentido el Proyecto Europeo DEPIT supone la creación y experimentación de un recurso tecnológico ideado para facilitar la programación docente desde una perspectiva inclusiva y personalizada. 


\section{Colás-Bravo, P.; Rossi, P. G.; de Pablos-Pons, J.; Conde-Jiménez, J. y Villaciervos Moreno, P. Aplicaciones digitales para la inclusión. El Proyecto Europeo DEPIT}

\subsection{El Proyecto Europeo DEPIT. Modelo pedagógico de referencia}

El proyecto europeo identificado con el acrónimo DEPIT (Designing for Personalization and Inclusion with Technologies), desarrollado desde 2017 a 2020, es un ejemplo de sinergia entre la práctica docente, la investigación educativa y la innovación didáctica. Es un proyecto cofinanciado por el programa Erasmus+ de la Unión Europea, en el que participan diferentes universidades europeas (Università di Macerata de Italia; Universidad de Sevilla de España; University College London de Reino Unido y Università Cattolica del Sacro Cuore de Italia), asociaciones internacionales de docentes y formadores (Centro del Profesorado Sevilla de España y Asociación AEDE-IT de Italia); redes de escuelas relacionadas con proyectos de innovación didáctica (Asociación Red DEPIT y Consorcio Red CRESCENDO de Italia); y empresas de desarrollo de software (Infofactory SRL de Italia). Todos ellos aportan distintas competencias fundamentales para alcanzar los objetivos marcados.

Su finalidad principal es desarrollar e implementar en los colegios una metodología didáctica emergente basada en la inclusión y la personalización de la enseñanza y proporcionar al profesorado una herramienta digital (la APP DEPIT) creada para facilitarles la labor de diseño y planificación de su docencia, a la vez que permitir a los estudiantes obtener una visión clara de sus itinerarios individualizados de aprendizaje. De modo que, el objetivo general de este proyecto es desarrollar, probar y transferir una metodología y una aplicación interactiva de diseño de aprendizaje en los sistemas escolares y universitarios participantes de los países socios, y luego promover su adopción en otros países de la Unión Europea a través de una APP.

Los objetivos específicos de este Proyecto se concretan en:

1. Desarrollar una metodología de planificación docente que apoye a los maestros en la atención tanto a realidades de aula diversas y complejas, como a las diferentes necesidades de los alumnos.

2. Crear una aplicación digital (para PC y móvil) sencilla e intuitiva y que se pueda usar tanto en el aula como en casa, para ayudar al profesorado a diseñar sus actividades de aprendizaje y así nutrir la programación.

3. Experimentar y validar esta herramienta digital (DEPIT) en diferentes niveles educativos, en los países que participaban en el proyecto.

4. Difundir y promover el uso de esta herramienta a través de la implementación de formación online abierta y masiva (MOOC).

5. Transferir esta aplicación y sus resultados a espacios formativos a nivel local, nacional y europeo.

La referencia teórica del modelo didáctico de DEPIT se basa en la teoría del "Conversational Framework" de la profesora Diana Laurillard (2012) que, teniendo en cuenta los resultados recientes de la investigación en el campo de la enseñanzaaprendizaje con TIC, entiende la figura del docente como un profesional de la planificación que necesita ayuda pedagógica y soporte digital para construir un ambiente o entorno de aprendizaje que le permita manejar y aprender con las tecnologías de una forma adaptativa. 


\section{Colás-Bravo, P.; Rossi, P. G.; de Pablos-Pons, J.; Conde-Jiménez, J. y Villaciervos Moreno, P. Aplicaciones digitales para la inclusión. \\ El Proyecto Europeo DEPIT}

Este entorno tecnológico de aprendizaje juega un papel crucial en la motivación del profesorado y del alumnado. Por un lado, actúa como guía para la organización y gestión didáctica del docente y, por otro, orienta la gestión curricular del estudiante. Los beneficios se concretan en ahorro de tiempo y recursos a la institución, aportar espacios interactivos de aprendizaje y promover el uso de las tecnologías en las aulas, sobre todo en el profesorado sin amplia formación en TIC.

Este enfoque aboga además por la colaboración entre docentes en la elaboración de los contenidos didácticos, permitiendo los procesos de reflexión, comparación, discusión y adaptación de las prácticas educativas y promueve un rol activo de los estudiantes en su proceso de aprendizaje, favoreciendo una enseñanza individualizada en beneficio del alumnado con diferentes habilidades.

En la siguiente ilustración podemos ver un resumen de las principales características y beneficios que la profesora Laurillard otorga a la creación de estos entornos tecnológicos de aprendizaje.

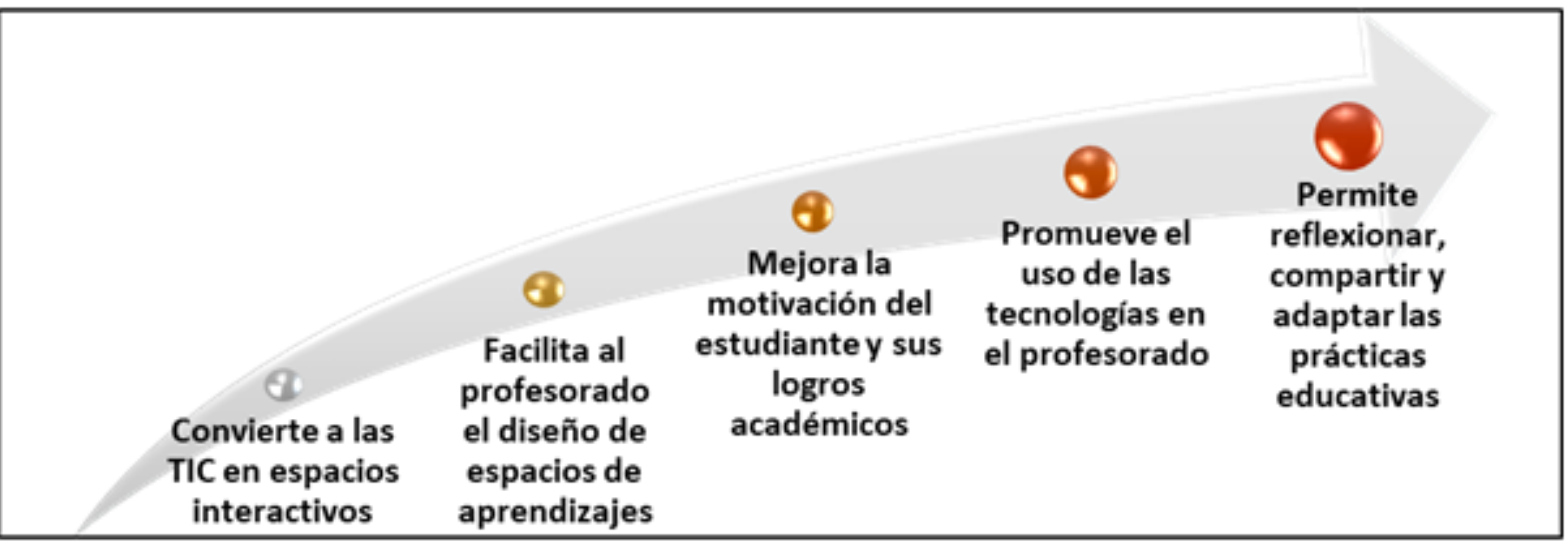

Imagen 1: Características de los Entornos Tecnológico de Aprendizaje según Laurillard et al. (2011).

Fuente: Elaboración propia, 2019.

El proyecto DEPIT conlleva las siguientes fases de actuación:

1. Primera. Fase de Diseño Metodológico: se definió y ajustó el enfoque metodológico en el que se fundamentaba el proyecto.

2. Segunda. Fase de Diseño y Pilotaje de la Herramienta: se desarrolló la aplicación para la planificación del aprendizaje con base al modelo pedagógico de referencia y se probó o testeó en los colegios y con los profesores pertenecientes a las redes y asociaciones participantes en el proyecto.

3. Tercera. Formación del Profesorado: se realizó el proceso de capacitación del profesorado para consolidar el uso de la Metodología y de la Aplicación. Esta formación se desarrolló a través de diferentes estrategias:

- Formación de los docentes de las asociaciones y redes participantes en el proyecto: curso de capacitación para los docentes de los colegios involucrados en la fase piloto. 
Colás-Bravo, P.; Rossi, P. G.; de Pablos-Pons, J.; Conde-Jiménez, J. y Villaciervos Moreno, P. Aplicaciones digitales para la inclusión.

\section{El Proyecto Europeo DEPIT}

- Formación a los futuros docentes y formadores: introducción de contenido específico sobre la metodología y aplicación DEPIT en algunas materias afines de titulaciones universitarias. Se incluyó este contenido en los planes de estudio de las titulaciones de educación de las Universidades participantes en el proyecto.

- Formación online, masiva y abierta: desarrollo del primer MOOC (curso a distancia, gratuito, accesible por internet al que se podía apuntar cualquier persona y prácticamente sin límite de participantes). Este curso constituyó el primero de una serie de MOOC ya planificados, que garantizaban el acceso directo y permanente a la metodología y a la APP DEPIT a otros colegios, universidades, docentes, estudiantes y cualquier otra persona interesada.

4. Cuarta. Transferencia y Generalización de Resultados: finalmente se destinó la última etapa a la divulgación de los resultados del proyecto a nivel local, nacional y europeo. Esta actuación supone la publicación de monográficos, comunicaciones, 0 artículos en revistas especializadas; la asistencia a congresos o seminarios relacionados con la temática; y la organización de eventos de difusión científica.

\subsection{La Herramienta Digital DEPIT}

La APP DEPIT constituye un recurso tecnológico novedoso diseñado para facilitar al profesorado el proceso de planificación docente. Esta planificación orientada al desarrollo de proyectos formativos se realiza tanto a nivel macro, como a nivel micro:

1. Macro: La planificación anual que el profesor decide a comienzo de año. Aquí es donde el docente describe el camino a seguir de cada asignatura y para todo su alumnado. Esta planificación se desarrolla a lo largo del curso académico.

2. Micro: La planificación de unidades didácticas o de cada sesión de trabajo que describe las actividades relacionadas con las diferentes clases sobre un mismo tema.

Los profesores necesitan, por tanto, organizar el contenido, temáticas, actividades y tareas diferentes y progresivas antes de que comiencen sus clases, es la antesala a la actividad educativa. Esta tarea de planificación concibe la actuación docente no como un conjunto de acciones imprevisibles y desconectadas entre sí, sino como la puesta en práctica de un plan bien pensado y articulado. Es justamente por eso que planificar es uno de los compromisos más importantes que debe asumir el profesorado, y se ha convertido en la actualidad en un proceso altamente cualificado de diseño pedagógico, sustentado en la producción de material didáctico bien anclado, ordenado y útil, no sólo para la clase en general, sino también adaptado a grupos concretos y necesidades específicas.

Esta responsabilidad del profesorado en particular y de la escuela de manera general, avala la importancia de brindarles toda la ayuda que desde las instituciones sea posible, y este es el compromiso que se asume desde el proyecto DEPIT, dotar al 


\section{Colás-Bravo, P.; Rossi, P. G.; de Pablos-Pons, J.; Conde-Jiménez, J. y Villaciervos Moreno, P. Aplicaciones digitales para la inclusión. El Proyecto Europeo DEPIT}

profesorado tanto de estrategias didácticas, como de recursos multimedia que favorezcan nuevas formas de diseñar, gestionar y organizar la enseñanza y posibiliten la creación de itinerarios personalizados y materiales educativos adaptados a las necesidades particulares de los estudiantes.

La aplicación DEPIT, que viene a cubrir estas necesidades, es un instrumento flexible, que facilita tanto la planificación de la enseñanza, como su posterior reestructuración durante la acción didáctica. El diseño pedagógico creado por los docentes es representado gráficamente mediante una estructura clara y visual que adopta la forma de itinerarios formativos paralelos o rutas de aprendizaje personalizadas. Para facilitar su uso y difusión, estas estructuras pueden visualizarse desde cualquier PC, Pizarra Digital Interactiva (PDI), o dispositivo móvil (Tablet o Smartphone).

\subsubsection{Funcionalidades de la APP DEPIT}

El principal propósito de DEPIT es ayudar al profesorado en su labor docente en cuanto a:

1. Facilitar al profesorado la labor de diseño de itinerarios de aprendizajes (programación didáctica) de manera flexible, inclusiva y personalizada: La aplicación permite que el profesorado piense y haga una planificación integrada y global de todas las situaciones educativas presentes en el aula. Esta visión se concreta de manera realista en tiempo, espacio y recursos educativos adaptados a la variedad de procesos de enseñanza-aprendizaje (en adelante EA) que pueden darse.

2. Organizar y gestionar la programación didáctica creada en diferentes niveles de concreción curricular: La APP se concibe como un espacio donde el docente materializa sus diseños en diferentes niveles de profundización: puede realizar una programación general articulada en módulos y una más concreta a través de lecciones o unidades didácticas y actividades independientes. Permite, por tanto, una planificación y gestión eficiente de los procesos de EA que el profesorado diseña.

En la siguiente imagen mostramos una programación anual donde se observan los diferentes niveles curriculares organizados secuencialmente haciendo uso de desplegables que incluyen Carpetas, y Tarjetas de colores diferentes. 
Colás-Bravo, P.; Rossi, P. G.; de Pablos-Pons, J.; Conde-Jiménez, J. y Villaciervos Moreno, P. Aplicaciones digitales para la inclusión. El Proyecto Europeo DEPIT

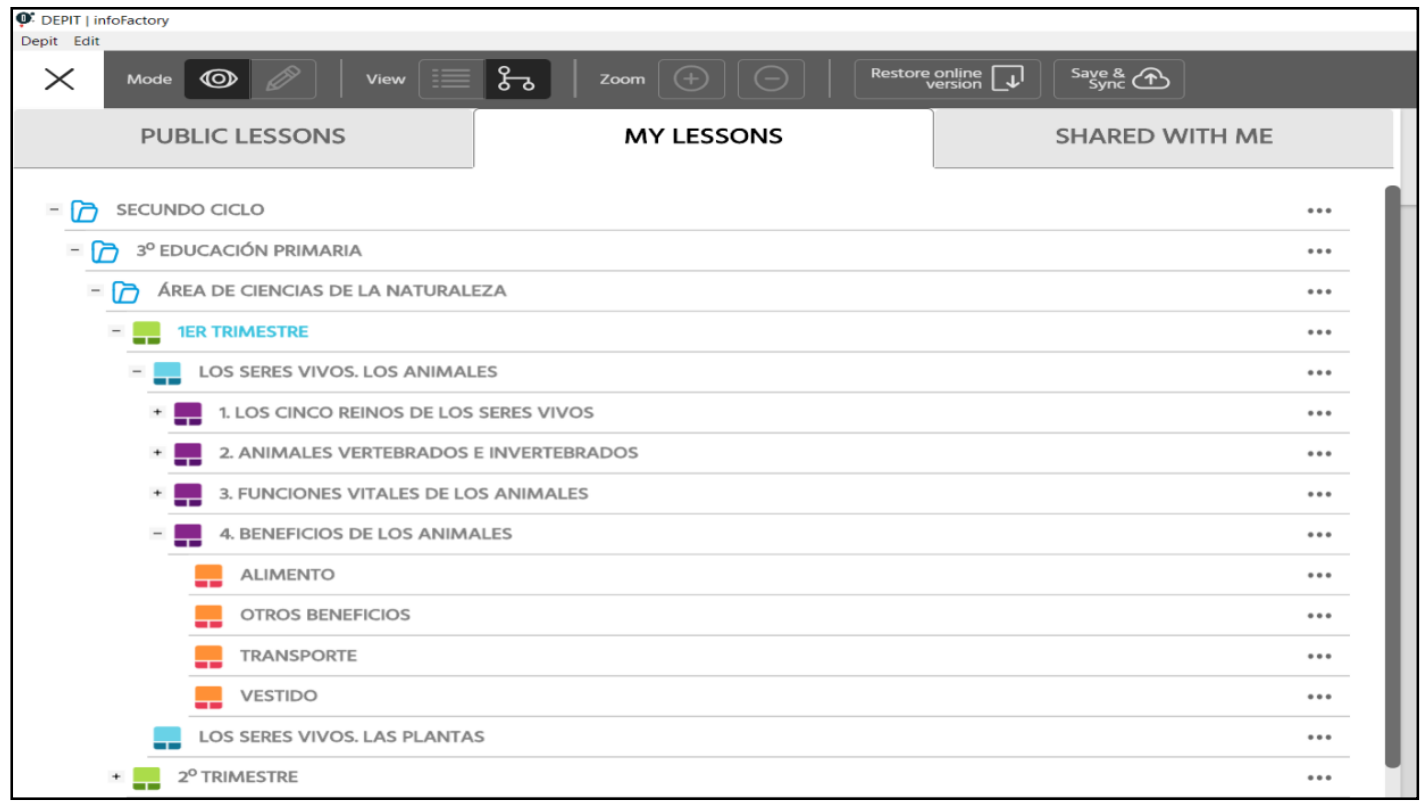

Imagen 2: Organización de la Programación Didáctica con la APP DEPIT.

Fuente: Elaboración propia, 2019.

En este caso se han generado distintas Carpetas para identificar el Ciclo, el Curso y el Área del currículum. Al mismo tiempo, se han utilizado Tarjetas para clasificar los contenidos por Trimestres (Tarjetas Verdes); por Módulos (Tarjetas Azules); y por Unidades Didácticas (Tarjetas Moradas). Por último, se han incorporado diferentes Actividades en cada Unidad (Tarjetas Naranjas).

3. Agregar recursos didácticos a la planificación docente: Como parte fundamental en la planificación docente, la herramienta permite al profesorado introducir diversidad de recursos en su programación didáctica para facilitar el proceso de aprendizaje de sus alumnos (textos, documentos, imágenes, vídeos, presentaciones, enlaces, etc.,). Estos materiales sirven también para captar la atención de los estudiantes y aumentar su motivación. Además, podrá personalizar cada uno de estos elementos, para hacer la propuesta lo más atractiva posible (cambiar imágenes de las tarjetas, insertar formas, introducir flechas para establecer conexiones, incluir fondos, etc.

4. Personalizar los procesos de EA (Enseñanza-Aprendizaje) para atender a la diversidad de los estudiantes: Desde la APP se pueden personalizar los itinerarios educativos y adaptar las actividades programadas a las diferentes capacidades o ritmos de aprendizaje que coexisten dentro de las aulas. Ello se realiza utilizando un código de colores que se pueden asignar a distintos niveles de dificultad de las actividades planteadas. También se contempla la posibilidad de rectificar el diseño didáctico en tiempo real, para adaptarlas a las necesidades del contexto concreto.

5. Integrar en el diseño de aprendizaje diferentes metodologías didácticas: La APP DEPIT permite incorporar metodologías didácticas diferentes ajustadas a las necesidades de los estudiantes y a los itinerarios educativos diseñados.

6. Gestión autónoma del aprendizaje por parte del estudiante en un entorno digital: DEPIT, al ser visible en el aula a través de proyectores o pizarras 
Colás-Bravo, P.; Rossi, P. G.; de Pablos-Pons, J.; Conde-Jiménez, J. y Villaciervos Moreno, P. Aplicaciones digitales para la inclusión.

\section{El Proyecto Europeo DEPIT}

digitales interactivas y estar disponible tanto para ordenadores (Mac y PC) como para dispositivos móviles (Tablet / Smartphone), permite que la planificación se haga explícita y abierta para ofrecer a los estudiantes información sobre su propio ritmo y proceso de aprendizaje. Se refuerza así en el docente un rol de guía de la formación y un papel activo del alumnado en su proceso de aprendizaje.

Una vez diseñada cada unidad didáctica, el estudiante puede gestionar su aprendizaje accediendo a los materiales diseñados y los recursos disponibles e interaccionar con el itinerario creado para trabajar lo que proceda en cada momento del proceso.

7. Compartir a nivel de centro y/o departamentos las unidades de aprendizaje creadas por el profesorado: Una de las problemáticas que afectan al profesorado actualmente es la falta de tiempo para coordinarse. Con DEPIT, existe la posibilidad de compartir el material didáctico que se elabore entre docentes. Ellos mismos pueden elegir invitar a los profesores y usuarios que estimen oportuno para compartir sus recursos propios, o bien, participar en la elaboración conjunta y simultánea de programaciones concretas. Al poner a disposición de los docentes la reposición y el intercambio de diseños didácticos, materiales y actividades educativas, se promueve la adopción de prácticas colaborativas en el profesorado generando una cultura de cooperación a nivel institucional.

8. Cohesionar las intenciones educativas del centro y la práctica docente: DEPIT favorece que las programaciones de aula estén armonizadas con las de centro favoreciendo la coordinación intra e interniveles, a la vez que permite la reflexión conjunta sobre los resultados del aprendizaje.

Una vez presentado el Proyecto DEPIT, en los apartados siguientes exponemos los resultados de la experimentación de esta herramienta tecnológica en las aulas de educación primaria y secundaria de España.

\section{OBJETIVOS}

La experimentación y validación aplicada de la herramienta digital (DEPIT) en diferentes niveles educativos, en los países que participaban en el proyecto, es uno de los objetivos del proyecto DEPIT. De ahí que en esta aportación presentemos el estudio llevado a cabo para aportar evidencias empíricas sobre el valor que le otorga el profesorado de España en sus funciones docentes.

Concretamente el objetivo de este estudio es conocer las actitudes del profesorado no universitario hacia el uso de aplicaciones TIC en la docencia, y en particular hacia el uso de la APP DEPIT en un colectivo de profesores españoles e identificar cómo puede ayudar la APP DEPIT a cubrir la necesidad de programación y diseño de los docentes, desde el punto de vista de estos.

\section{METODOLOGÍA}

Este estudio, utiliza un enfoque multimétodo, en tanto integra metodología cuantitativa y cualitativa. Se utiliza una metodología cuantitativa-descriptiva para 


\section{Colás-Bravo, P.; Rossi, P. G.; de Pablos-Pons, J.; Conde-Jiménez, J. y Villaciervos Moreno, P. Aplicaciones digitales para la inclusión. \\ El Proyecto Europeo DEPIT}

conocer las actitudes del profesorado hacia la APP DEPIT. Y una metodología cualitativa para identificar las necesidades de programación y diseño en los que la APP DEPIT puede ayudar a los docentes. Los datos cualitativos se obtienen de las aportaciones escritas vertidas en un foro por el profesorado que se ha formado y experimentado con DEPIT. Los datos cuantitativos se obtienen mediante una escala tipo Likert diseñada ad hoc para este estudio.

Los participantes en el estudio son docentes españoles que participaron en el testeo de la APP DEPIT. Estos pertenecen a centros seleccionados de forma intencional por el Centro de Profesorado (CEP) de Sevilla (España), siendo centros que estaban abiertos a participar en el estudio $y$, por otro lado, eran centros con una experiencia en colaboración con el CEP y con trayectoria en el uso y aplicación de las TIC. Además, este profesorado participó en el curso "Designing for Personalization and Inclusion with Technology (DEPIT)- Módulo 1" (Código: CEPSE1_184127ALI021) impartido por el CEP de Sevilla y diseñado por el Grupo de Investigación, Evaluación y Tecnología Educativa (HUM154) de la Universidad de Sevilla. Este módulo curso consta de dos horas presenciales y seis horas no presenciales, y se desarrolló desde el 26 de junio hasta el 13 de julio de 2018. Específicamente, participaron 23 profesores, de los que el $58,3 \%$ eran mujeres y el $41,7 \%$ hombres, situados en un rango de edad que oscilaba desde los 29 a los 56 años. Todos tenían más de 5 años de experiencia docente. El $75 \%$ en Educación Primaria y el otro $25 \%$ en Enseñanza Secundaria, Bachillerato o Formación Profesional. En una escala de 1 a 10, siendo 1 la mínima puntuación y 10 la máxima, el $70 \%$ del profesorado considera que tiene un nivel de competencia digital por encima de 8 . Y el $75 \%$ participa en algún grupo de trabajo o innovación en su centro.

Para el objetivo 1 se ha elaborado una escala ad hoc, integrada por 15 ítems, de los cuales 8 preguntan sobre la actitud del profesorado sobre el uso de aplicaciones TIC para programar la docencia y 7 ítems referidos específicamente a la aplicación de APP DEPIT. En la siguiente Imagen 9, se puede ver la relación de ítems de la escala, en la que aparecen con asterisco aquellas actitudes hacia el uso de las TIC en general, y sin asterisco hacia la usanza específica de la APP DEPIT.

Tabla 1. Escala de Actitudes hacia el uso de aplicaciones TIC en el aula.

\begin{tabular}{|c|}
\hline $\begin{array}{c}\text { En general, me siento seguro sobre mi capacidad para utilizar aplicaciones informáticas para diseñar } \\
\text { programaciones de aula* }\end{array}$ \\
\hline Me genera estrés el uso de ordenadores* \\
\hline El desafío de utilizar aplicaciones informáticas para la actividad docente es emocionante* \\
\hline Cualquiera puede aprender a usar una aplicación informática si es paciente y está motivado* \\
\hline $\begin{array}{c}\text { Prefiero hacer las cosas de forma tradicional, en vez de tener que usar una aplicación informática } \\
\text { nueva* }\end{array}$ \\
\hline Me temo que, si empiezo a utilizar aplicaciones informáticas, seré más dependiente de ellas y \\
perderé algunas de mis habilidades de razonamiento*
\end{tabular}


Colás-Bravo, P.; Rossi, P. G.; de Pablos-Pons, J.; Conde-Jiménez, J. y

Villaciervos Moreno, P. Aplicaciones digitales para la inclusión.

El Proyecto Europeo DEPIT

\begin{tabular}{|c|}
\hline Tengo dificultades para entender los aspectos técnicos de la aplicación DEPIT \\
\hline $\begin{array}{c}\text { Me asusta pensar que podría perder el trabajo y una gran cantidad de información por un fallo de la } \\
\text { aplicación DEPIT }\end{array}$ \\
\hline Pienso que utilizar la aplicación DEPIT me ahorrará mucho trabajo tedioso a la hora de programar \\
\hline Considero que el uso de la aplicación DEPIT me generará frustración \\
\hline Pienso que el uso de la aplicación DEPIT mejorará mi actividad docente \\
\hline 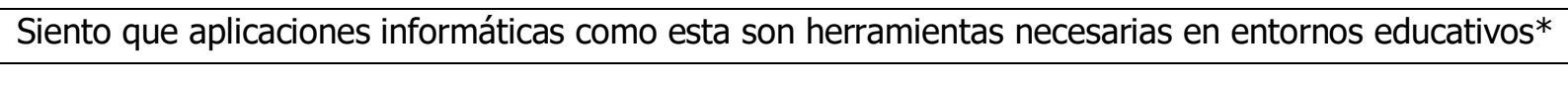 \\
\hline
\end{tabular}

Fuente: Elaboración propia, 2019.

Se pide que cada una de estas afirmaciones se valoren de 1 a 10, según el nivel de acuerdo con las mismas, siendo $1=$ Nada De Acuerdo y $10=$ Completamente De Acuerdo.

Para dar respuesta al objetivo 2, se utiliza un foro integrado en el curso realizado en el CEP por el profesorado, en dicho espacio se les insta a los docentes que expongan, debatan y discutan de forma interactiva sobre los aspectos potenciales de la aplicación DEPIT para la programación de la enseñanza.

Para el análisis de datos de los resultados cuantitativos derivados de la aplicación de la escala, se usa el programa SPSS V.24, para realizar descriptivos estadísticos de frecuencias (\%). Para el análisis estadístico de los datos cualitativos de los foros, se utiliza el Atlas.Ti V.8, siguiéndose el siguiente proceso metodológico:

1. Lectura de las aportaciones realizadas por los docentes en el foro.

2. Análisis previo e identificación de variables y/o categorías.

3. Codificación de las aportaciones asignando códigos a la narración.

4. Organización y estructuración de la información con el fin de realizar una construcción o elaboración teórica explicativa de las dimensiones emergidas.

\section{RESULTADOS}

En relación, al primer objetivo de este estudio, de conocer las actitudes del profesorado no universitario hacia el uso de aplicaciones, existen dos agrupaciones de respuestas. Por un lado, obtenemos respuestas positivas del profesorado en afirmaciones vinculadas a su capacidad para usar aplicaciones digitales para programar su propia enseñanza, la consideración de que la aplicación de herramientas es algo motivante y emocionante. Además, consideran que la aplicación de herramientas informáticas es necesaria en los entornos educativos y que cualquier docente puede aplicar, si es paciente ye está motivado. Con respecto a la APP DEPIT, están seguros de que con tiempo y práctica podrán sentirse cómodos al usarla, podrán seguir sus pasos para aplicarlas de forma adecuada, y que le ahorrará tiempo a la hora de realizar la tediosa tarea de programar, considerándose que mejorará la actividad docente. Estos resultados pueden visualizarse en el siguiente gráfico 1 , en el que puede observarse que la mayoría de los profesores se sitúa, más del $75 \%$, en valores positivos a la hora de estar de acuerdo con dichas afirmaciones. 


\section{Colás-Bravo, P.; Rossi, P. G.; de Pablos-Pons, J.; Conde-Jiménez, J. y Villaciervos Moreno, P. Aplicaciones digitales para la inclusión. El Proyecto Europeo DEPIT}

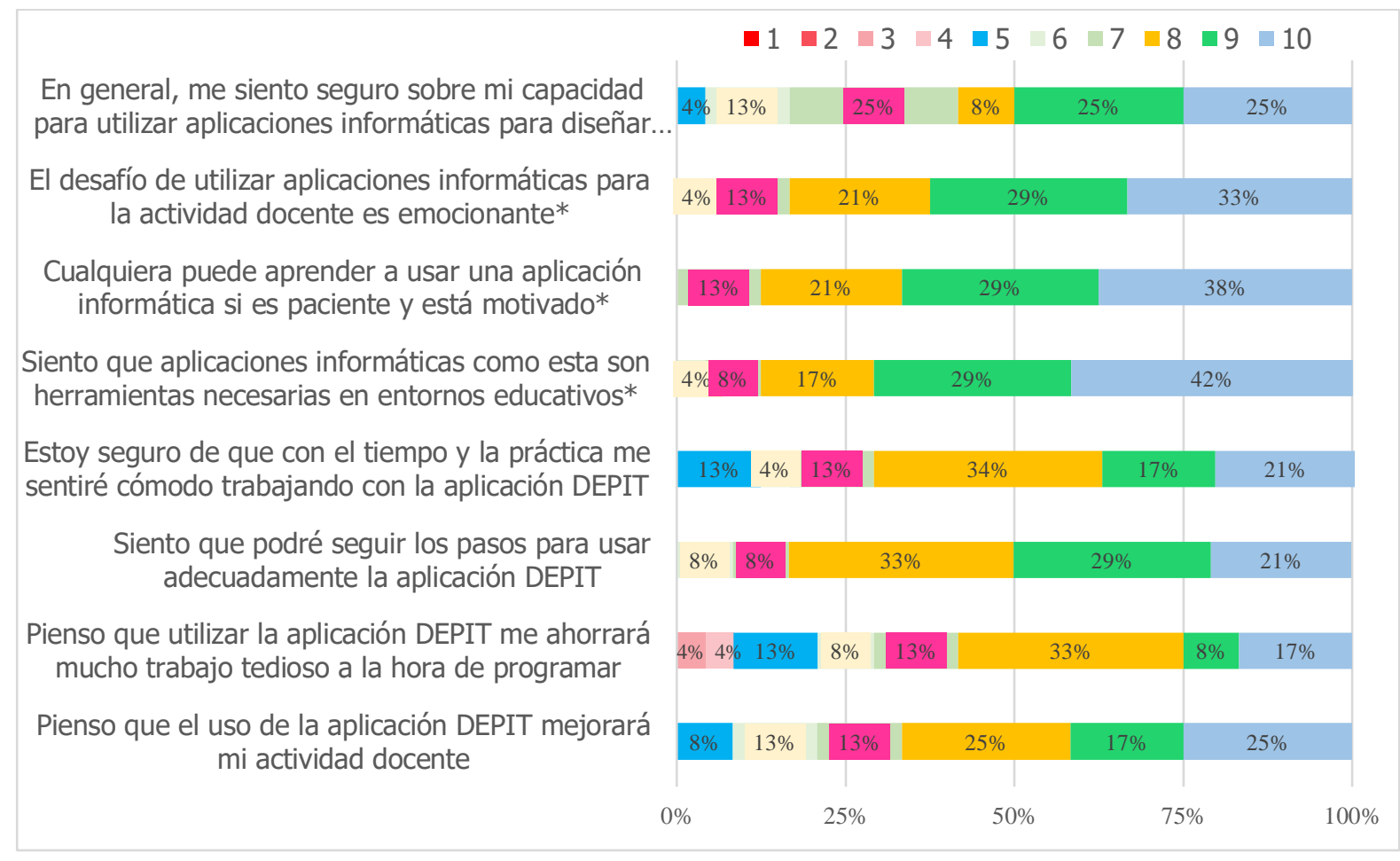

Gráfico 1: Valoraciones positivas hacia el uso de aplicaciones TIC y de la APP DEPIT.

Fuente: Elaboración propia, 2019.

Por en el contrario, en el siguiente gráfico 2, puede observarse aquellas afirmaciones con las que el profesorado está en desacuerdo. Puede detectarse cómo no tienen una actitud negativa hacia el uso de las TIC en la enseñanza, manifestando que consideran que el uso de ordenadores no les genera estrés, que no prefieren hacer las cosas de forma tradicional, sino que prefieren usar aplicaciones informáticas nuevas, como, por ejemplo, prefieren usar herramientas digitales para hacer programaciones. Además, no están de acuerdo con la afirmación de que, si hacen uso de aplicaciones informáticas, serán más dependientes de ellas y perderán habilidades de razonamiento. En cuanto a la APP DEPIT, tienen una actitud bastante abierta para su uso, ya que consideran que no tienen dificultades para entender esta y consideran que no les generará frustración.

Revista de Comunicación de la SEECI. 15 noviembre, 2019 / 15 marzo, 2020, n 50, 169-192 
Colás-Bravo, P.; Rossi, P. G.; de Pablos-Pons, J.; Conde-Jiménez, J. y

Villaciervos Moreno, P. Aplicaciones digitales para la inclusión. El Proyecto Europeo DEPIT

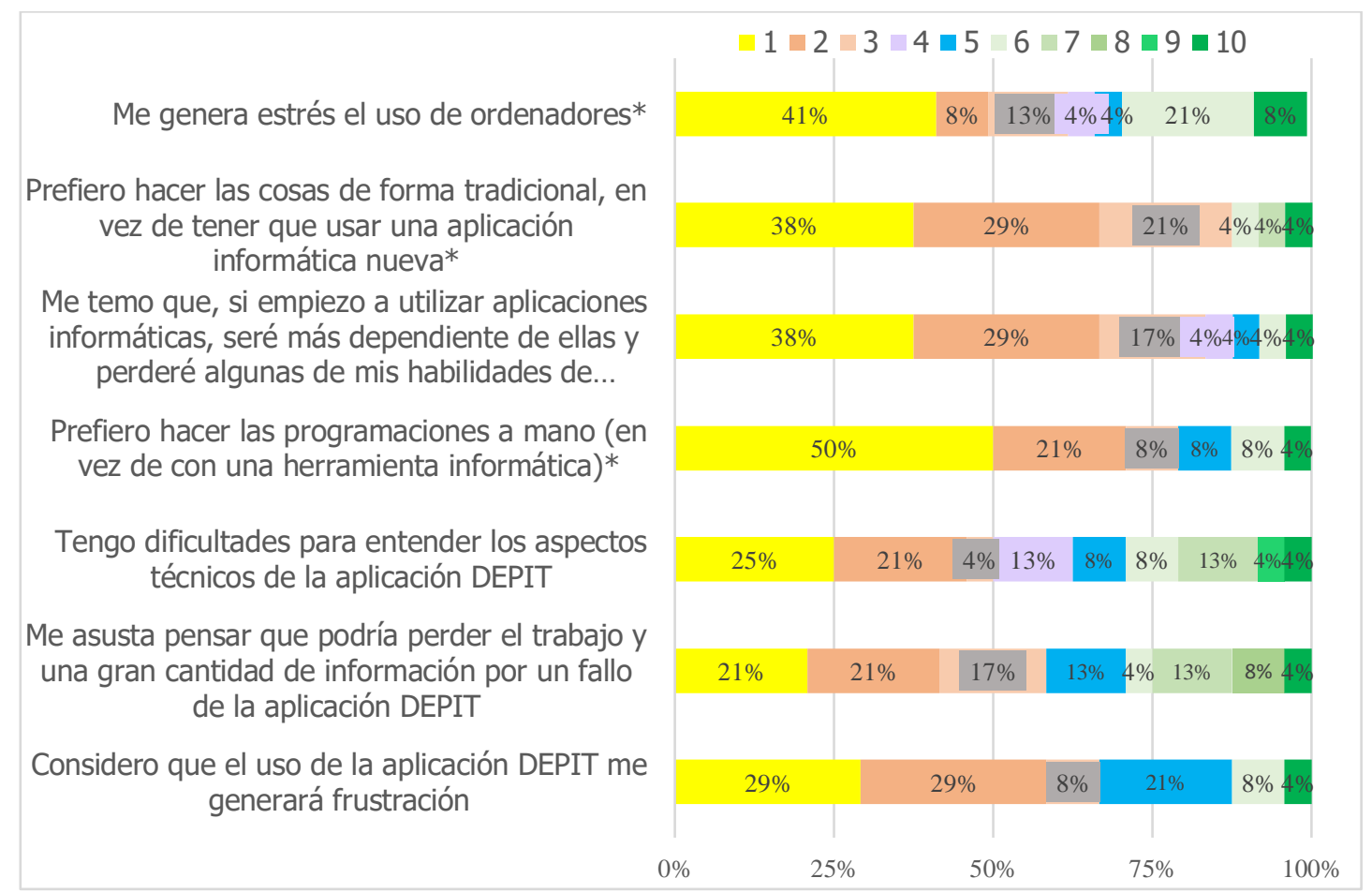

Gráfico 2: Valoraciones negativas hacia el uso de aplicaciones TIC y de la APP DEPIT.

Fuente: Elaboración propia, 2019.

Por lo que se puede apreciar en el gráfico 2, en sus niveles de respuesta se detecta una actitud bastante positiva para el uso de herramientas informáticas y digitales para realizar la programación docente, también de DEPIT. Ya que los mayores porcentajes de respuesta se concentran en una valoración baja de estos aspectos negativos.

El análisis cualitativo aplicado a las manifestaciones de los docentes, en los foros del curso de formación, posibilita identificar y extraer dimensiones en las que la herramienta digital APP DEPIT puede ayudar en la programación docente. Se observan y registran las siguientes dimensiones.

1. Dimensión 1. Adaptación y versatilidad para contextos educativos: el profesorado que ha testado la aplicación, considera que esta tiene potencial para ser transferida y ajustada a distintos contextos escolares, principalmente debido a su versatilidad, facilidad de uso, acceso e inclusión de múltiples recursos... lo que le permite aplicarse a la tarea de programar la enseñanza y personalizarla según las distintas características de la clase. Algunas citas que respaldan esta dimensión son:

La app del proyecto DEPIT, desde mi punto de vista, es una herramienta muy versátil. Se puede adaptar sin demasiadas dificultades a la programación por unidades didácticas integradas. (Sujeto 1)

Revista de Comunicación de la SEECI. 15 noviembre, 2019 / 15 marzo, 2020, n 50, 169-192 
Colás-Bravo, P.; Rossi, P. G.; de Pablos-Pons, J.; Conde-Jiménez, J. y Villaciervos Moreno, P. Aplicaciones digitales para la inclusión.

\section{El Proyecto Europeo DEPIT}

Una APP, en concreto la del proyecto DEPIT, puede ayudar a facilitar nuestra programación ya que nos permite acceder a los recursos desde cualquier aparato tecnológico tanto al profesorado como al alumnado, proporcionado mayor motivación e interés, facilitando la participación y sobre todo porque da la opción de tener en cuenta la diversidad de nuestro alumnado. (Sujeto 6)

Posiblemente una app puede apoyar positivamente nuestra programación porque debe ser abierta y se adaptará al alumnado, a su contexto, a sus dificultades, a la variedad de nuestros niños y niñas, e incluso a las diferentes formas de docencia que podemos encontrar en nuestros coles. (Sujeto 3)

La utilidad de una APP es que nos permite adaptar la programación a las necesidades de nuestra aula, permitiéndonos personalizarla y realizar un diseño inclusivo. (Sujeto 8)

Posiblemente una app puede apoyar positivamente nuestra programación porque debe ser abierta y se adaptará al alumnado, a su contexto, a sus dificultades, a la variedad de nuestros niños y niñas, e incluso a las diferentes formas de docencia que podemos encontrar en nuestros coles. (Sujeto 3)

2. Dimensión 2. Facilita la interacción y la comunicación: los docentes destacan que al ser tan accesible y permitir que profesorado y alumnado trabajen en el mismo espacio se amplían las oportunidades interactivas entre ambos agentes. Además, es una plataforma que permite la comunicación síncrona y asíncrona. También, destacan que se expanden y se transciende a los propios límites del aula, y la se incluye en esta a otros agentes de la comunidad educativa.

Es un nuevo canal de comunicación, más rápido y directo, además de accesible a todos... Información accesible a todos los miembros de la comunidad educativa. (Sujeto 2)

Sin embargo, me llama la atención de la que vamos a trabajar en concreto, el hecho de poder compartirla con el alumnado, que ellos vean nuestra propia programación que, al fin y al cabo, va a recaer sobre ellos. Es la programación de la enseñanza para su propio aprendizaje, así que, si lo podemos compartir con ellos, mejor que mejor. (Sujeto 5)

Además, permitirá la buena comunicación entre todos los miembros de la comunidad educativa que, bajo mi punto de vista, es fundamental. (Sujeto 7)

Es un canal de comunicación abierto a toda la comunidad, nos permite acceder a los enlaces y material de forma sencilla. (Sujeto 9)

3. Dimensión 3. Ventajas relativas al diseño y características de DEPIT: el propio diseño de la APP es marcado como un potencial. Además, la funcionalidad de permitir la incorporación de diversos recursos es señalado como una característica positiva. 
Colás-Bravo, P.; Rossi, P. G.; de Pablos-Pons, J.; Conde-Jiménez, J. y Villaciervos Moreno, P. Aplicaciones digitales para la inclusión.

\section{El Proyecto Europeo DEPIT}

Visualmente, es atractiva y muy adecuada para trazar diferentes itinerarios dentro de la propuesta didáctica, que se amolden a los distintos ritmos de aprendizaje. (Sujeto 1)

Algunas de las características y utilidades que debe tener la APP a mi juicio son: Mostrar los contenidos y actividades que planteas permitiendo añadirlos de una forma sencilla y en una gran variedad de formatos; permitir también realizar una jerarquía de las unidades, temas y actividades, de forma que sea sencillo localizar "donde estamos"; ayudar a nuestros estudiantes a comprender la estructura de los contenidos y unidades. Creo que este es uno de los aspectos interesantes de esta aplicación, su carácter visual en contraposición con las otras aplicaciones que he usado que tienen un carácter más lineal. (Sujeto 8)

Fácil de manejar... se visualiza globalmente los diferentes elementos... Flexible y adaptable a cambios. (Sujeto 11)

Al poder añadirse recursos en todos los niveles, resulta muy práctica y motivadora. (Sujeto 1)

Como el alumnado también tendrá acceso, servirá para controlar su trabajo y siempre se tendrán a mano los recursos (imágenes, webs, vídeos, archivos, etc.). (Sujeto 1)

Posibilidad de acceder a los recursos desde cualquier aparato tecnológico (ordenador, Tablet, móvil,) y en cualquier lugar. (Sujeto 2)

4. Dimensión 4. Se potencia la libertad, independencia y autonomía: en relación a lo anterior, la propia concepción y diseño de la APP hace que los docentes sean agentes constructores de todo el proceso de enseñanzaaprendizaje, modificándose y adaptándose siempre que sea necesario:

Otro aspecto interesante de la APP es que nos ayuda a los docentes a que seamos los "directores de orquesta", no dejándole esa función a ninguna editorial concreta. Nosotros debemos ser los que programemos en función de nuestro alumnado. (Sujeto 1)

5. Dimensión 5. Se consigue la personalización: con base a todas las dimensiones anteriores los docentes consideran que esta aplicación permite la personalización de la enseñanza:

Una atención más especializada con vínculos a diversos recursos. (Sujeto 4)

Se adapta al dinamismo de las unidades didácticas y, por ende, a la inclusión. (Sujeto 7) 
Colás-Bravo, P.; Rossi, P. G.; de Pablos-Pons, J.; Conde-Jiménez, J. y Villaciervos Moreno, P. Aplicaciones digitales para la inclusión.

El Proyecto Europeo DEPIT

La utilidad de una APP es que nos permite adaptar la programación a las necesidades de nuestra aula, permitiéndonos personalizarla y realizar un diseño inclusivo. (Sujeto 8)

6. Dimensión 6. Desarrollo de la competencia digital: el uso de la herramienta fomenta el entrenamiento y perfeccionamiento de la competencia digital:

Mejora la competencia digital en la era de las nuevas tecnologías. (Sujeto 2)

Por último, fomenta la competencia digital $y$, por supuesto, siempre es positivo tener una herramienta de apoyo. (Sujeto 7)

Intentamos trabajar las TIC y una aplicación de este tipo nos puede servir sobremanera en ofrecer a nuestro alumnado una visión de nuestra asignatura mucho más atractiva, visual, virtual, adaptada a su forma ver el mundo que les rodea. (Sujeto 10 )

7. Dimensión 7. Mejora la motivación y el engagement. el profesorado considera que el uso de DEPIT puede ayudar tanto a profesorado como al alumnado a un mayor motivación e implicación con lo curricular:

Mayor motivación hacia los aprendizajes al tener tanto alumnado como profesorado un rol activo y conectividad entre todos, dentro y fuera del centro educativo. (Sujeto 2)

La APP del proyecto DEPIT, supone en primer lugar una novedad. Las novedades siempre consiguen mantener a nuestros alumnos y alumnas expectantes y abiertos al nuevo aprendizaje. (Sujeto 7)

...cuenta con "la novedad", por lo que la motivación del alumnado está garantizada. (Sujeto 9)

\section{DISCUSIÓN}

En cuanto al objetivo 1 del estudio, se puede concluir que los docentes tienen una actitud bastante positiva el uso de aplicaciones para la aplicación de la tarea docente de la programación. Igualmente, las actitudes hacia la APP DEPIT son igualmente positivas. Lo que evidencia una actitud abierta de los profesores hacia herramientas tecnológicas que les puedan ayudar en su labor profesional.

En cuanto al objetivo 2, respecto a la APP DEPIT, el profesorado reconoce el valor de esta en tanto que permite incluir multitud de recursos, su carácter interactivo y comunicativo, además de posibilitar su adaptación a múltiples contextos educativos.

El docente se percibe como agente transformador de cambio que se sirve de esta herramienta digital para mediar en la personalización de su enseñanza, siempre 


\section{Colás-Bravo, P.; Rossi, P. G.; de Pablos-Pons, J.; Conde-Jiménez, J. y Villaciervos Moreno, P. Aplicaciones digitales para la inclusión. \\ El Proyecto Europeo DEPIT}

ajustada a las características particulares de la propia aula. El desarrollo de la competencia digital es un aspecto potenciador, y muy en línea con las directrices europeas, marco DIGCOMPEDU, que insta a los docentes no sólo al entrenamiento de su propia competencia digital sino a la de sus estudiantes. Las distintas dimensiones emergidas permiten que el propio docente controle su propia docencia, pero también da la oportunidad de empoderar a sus propios estudiantes.

Desde un enfoque más amplio podemos también concluir que este proyecto DEPIT es una contribución a una educación sostenible, en tanto coadyuva a la inclusión y mejora educativa, además de reivindicar la individualidad. La posibilidad de reelaboración de las propuestas programáticas es otro aspecto destacable de la sostenibilidad, ya que una iniciativa sostenible es una propuesta que permite el aprovechamiento de los recursos, así como la posibilidad de permanencia y continuidad del trabajo inicialmente invertido. Para el profesorado un producto flexible es sostenible, en tanto es capaz de canalizar la creatividad y la posibilidad de ajustarse en la acción, intuitiva y racional, ya que incluso los posibles errores en la planificación didáctica pueden detectarse y por lo tanto eliminarse

Por tanto y concluyendo, los resultados experimentales de la aplicación DEPIT aquí presentados, aportan datos contrastados e indicativos del potencial de la APP DEPIT para una enseñanza planificada y sostenible.

\section{RECOMENDACIONES}

Esta investigación expone sólo los resultados de la investigación desarrollada con docentes españoles de enseñanza primaria y secundaria, queda por ver si estos hallazgos convergen con otros niveles universitarios y/o con el resto de los países participantes en el estudio. Por otro lado, es necesario experimentar y evaluar esta herramienta tecnológica en poblaciones más amplias de profesorado que tengan diferentes niveles competenciales en el uso de las TIC en sus aulas, así como diferentes trayectorias formativas y de conocimientos. El carácter europeo de este proyecto ofrece la oportunidad de contar con información procedente de profesorado de diferentes niveles y procedencias geográficas para poder llegar a conclusiones más extensivas y generales.

\section{REFERENCIAS}

Bauman, Z. (2018). Generación líquida: Transformaciones en la era 3.0. Barcelona: Editorial Planeta.

Berthoz, A. (2011). Simplexité. Paris: Odile Jacob.

Bosada, M. (2018). El reto de personalizar el aprendizaje con ayuda de las TIC, Educaweb. Recuperado de https://www.educaweb.com/noticia/2018/05/31/retopersonalizar-aprendizaje-ayuda-tic-18489/ 
Colás-Bravo, P.; Rossi, P. G.; de Pablos-Pons, J.; Conde-Jiménez, J. y Villaciervos Moreno, P. Aplicaciones digitales para la inclusión.

El Proyecto Europeo DEPIT

Caminal, A. y Puigcerver, M. (2017). Diseños de campus virtual: descripción de la oferta actual en la enseñanza de las ciencias experimentales y su influencia en el uso por parte del alumnado universitario, Diddacticae, (2), 119-133. doi: https://doi.org/10.1344/did.2017.2.119-133

Cubeles, A. \& Riu, D. (2018). The effective integration of ICTs in universities: the role of knowledge and academic experience of professors, Technology, Pedagogy and Education, 27(3), 339-349.

doi: https://doi.org/10.1080/1475939X.2018.1457978

De Pablos, J.; Colás, P.; López, A. \& García, I. (2019). Uses of digital platforms in Higher Education from the perspectives of the educational research, REDU. Revista de Docencia Universitaria, 17(1), 59-72.

doi: https://doi.org/10.4995/redu.2019.11177

Dewey, J. (1938). Experience and Education. New York: Kappa Delta Pi.

Fernández, I. (2017). Mejora de competencias: Introducción de la gestión de calidad en nuevas metodologías educativas, Revista de Currículum y Formación del Profesorado, 21(2), 279-308. Recuperado de https://recyt.fecyt.es/index.php/profesorado/article/view/59461/36173

Gámiz, V. y Gallego, M. J. (2016). Modelo de análisis de metodologías didácticas semipresenciales en Educación Superior, Educación XX1, 19(1), 39-61. doi: https://doi.org/10.5944/educxx1.15577

Grande, P. y González, M. M. (2015). La educación inclusiva en la educación infantil: propuestas basadas en la evidencia. Tendencias Pedagógicas, (26), 145-162. Recuperado de https://dialnet.unirioja.es/descarga/articulo/5247176.pdf

Laurillard, D. (2012). Teaching as design science. New York: Routdledge.

Laurillard, D. et al. (2011). A constructionist learning environment for teachers to model learning designs, Journal of Computer Assisted Learning, 29(1), 15-30. doi: https://doi.org/10.1111/j.1365-2729.2011.00458.x

Molano, E. G.; Sánchez, O. N. y Castillo, J. N. (2012). Aprendizaje con modelo TCPK y e-learning de la lógica de programación, Revista vínculos, 9(2), 57-72. doi: https://doi.org/10.14483/2322939X.4287

Onrubia, J. (2016). Aprender y enseñar en entornos virtuales: actitud conjunta, ayuda pedagógica y construcción del conocimiento, RED. Revista de Educación a Distancia, 50(3), 1-14. doi: https://doi.org/10.6018/red/50/3

Organización de Estados Iberoamericanos (OEI). Inclusión Educativa. Recuperado de https://oei.cl/inclusion-educativa/inclusion-educativa 


\section{Colás-Bravo, P.; Rossi, P. G.; de Pablos-Pons, J.; Conde-Jiménez, J. y Villaciervos Moreno, P. Aplicaciones digitales para la inclusión.

Pineda, P. y Castañeda, A. (2013). Comunicación dialógica y ciberparlamentos españoles, Sphera Pública, junio, 44-63. Recuperado de: http://sphera.ucam.edu/index.php/sphera-01/article/view/197/168

Siu, K. W. \& Lam, M. S. (2012). Public computer assisted learning facilities for children with visual impairment: Universal design for inclusive learning. Early Childhood Education Journal, 40(5), 295-303.

Touron, J. (2019). Aprendizaje personalizado y tecnología. [Mensaje en un blog]. Recuperado de https://www.javiertouron.es/aprendizaje-personalizado-tecnologia/

UNESCO (2016). Educación 2030: Declaración de Incheon y Marco de Acción para la realización del Objetivo de Desarrollo Sostenible 4. Recuperado de https://unesdoc.unesco.org/ark:/48223/pf0000245656 spa

\section{AUTORES:}

\section{Pilar Colás Bravo}

Catedrática de Métodos de Investigación y Diagnóstico en Educación. Imparte docencia en la Universidad de Sevilla (España). Miembro fundador del grupo de Investigación, Evaluación y Tecnología Educativa (GIETE). Ha liderado numerosos Proyectos de Investigación nacionales e internacionales y Proyectos de Innovación originando patentes. Sus líneas de investigación son: Investigación Educativa y TIC, Género, Diversidad e Inclusión, Evaluación y Calidad Educativa. Autora de más de un centenar de publicaciones en revistas de impacto. Autora y editora de libros y revistas científicas de impacto. Colabora con la Agencia Nacional de Evaluación de la Calidad y la Acreditación (ANECA) y con la Agencia Nacional de Evaluación y Prospectiva (ANEP). También colabora en calidad de Experta en otras agencias internacionales.

pcolas@us.es

Orcid ID: https://orcid.org/0000-0003-3000-075X

Google Scholar: https://scholar.google.com/citations?user=fjPPRJQAAAAJ\&hl=es

ResearchGate: https://www.researchgate.net/profile/Pilar Bravo2

Academia.edu: https://us.academia.edu/PilarColasBravo

Researcher ID: http://www.researcherid.com/rid/D-5889-2017

Publons: https://publons.com/researcher/2143710/pilar-colas/

\section{Pier Giuseppe Rossi}

Catedrático de Didáctica General y Pedagogía de Necesidades Educativas Especiales, en el Departamento de Ciencias de la Educación, Patrimonio Cultural y Turismo de la Universidad de Macerata (Italia). Decano de la Facultad de Educación desde 2009 hasta 2012, y miembro de la Junta de Directores de la Universidad de Macerata desde 2012 hasta 2016. Actualmente es delegado de informática, innovación didáctica y e-learning en la Universidad de Macerata. Es presidente de SIREM (Sociedad Italiana de Investigación en Educación Mediática) y miembro de comités científicos de revistas de impacto y colecciones editoriales. Ha coordinado más de 10 


\section{Colás-Bravo, P.; Rossi, P. G.; de Pablos-Pons, J.; Conde-Jiménez, J. y Villaciervos Moreno, P. Aplicaciones digitales para la inclusión. \\ El Proyecto Europeo DEPIT}

proyectos europeos. Ahora coordina el proyecto europeo DEPIT. Y cuenta con más de 200 publicaciones (libros, artículos y capítulos de libros).

piergiuseppe.rossi@unimc.it

Orcid ID: https://orcid.org/0000-0001-9801-6307

Google Scholar: https://scholar.google.com/citations?user=-Aiv3BEAAAAJ\&hl=it

ResearchGate: https://www.researchgate.net/profile/Pier Giuseppe

Academia.edu: https://unimc.academia.edu/PRossi

ResearcherID: http://www.researcherid.com/rid/H-7776-2015

\section{Juan de Pablos Pons}

Catedrático de Universidad desde el año 1995 en el área de Didáctica y Organización Escolar, en la Universidad de Sevilla. Director del Grupo de Investigación Evaluación y Tecnología Educativa, vinculado al Plan Andaluz de Investigación. Es autor de numerosas publicaciones $y$ ha participado con regularidad en proyectos de investigación subvencionados, a nivel autonómico, nacional y europeo. Evaluador de proyectos I+D de distintas agencias nacionales y autonómicas. Las líneas de investigación preferentes, desarrolladas durante los últimos diez años han sido: Las TIC aplicadas a la educación; las políticas educativas y la integración de las TIC; la formación del profesorado; y el desarrollo del Espacio Europeo de Educación Superior. En el ámbito de la gestión, ha desempeñado el puesto de Decano de la Facultad de Ciencias de la Educación desde el año 2009 hasta el 2017; ha sido presidente de la Asociación Científica Red Universitaria de Tecnología Educativa (RUTE).

jpablos@us.es

Orcid ID: https://orcid.org/0000-0001-9147-5965

Google Scholar: https://scholar.google.com/citations?user=HIOusEkAAAAJ

ResearchGate: https://www.researchgate.net/profile/Juan Pons2

Academia.edu: https://us.academia.edu/Juan+de+Pablos+Pons

ResearcherID: http://www.researcherid.com/rid/K-9981-2014

Publons: https://publons.com/researcher/2489576/juan-de-pablos-pons/

\section{Jesús Conde-Jiménez}

Doctor en Ciencias de la Educación, con mención Internacional, Máster en Dirección, Evaluación y Calidad de las Instituciones de Formación y Licenciado en Psicopedagogía por la Universidad de Sevilla (España). Actualmente, es profesor a tiempo completo en la Universidad Loyola Andalucía y es miembro del Grupo de Investigación, Evaluación y Tecnología Educativa (GIETE) HUM-154, desde el año 2011. Durante 2013-2017, he sido beneficiario de una ayuda predoctoral, de naturaleza competitiva, dentro del programa nacional de Formación del Profesorado Universitario (FPU) del Ministerio de Educación, Cultura y Deporte (España). Ha participado en distintos proyectos a nivel internacional y nacional. Cuenta con diversos artículos en revistas indexadas en JCR (Comunicar, Sustainability, Cultura y Educación...) y Scopus (en Bordón. Revista de Pedagogía o en la Revista Electrónica de Investigación Educativa).

jconde@uloyola.es

Orcid ID: https://orcid.org/0000-0002-4471-5089

Google Scholar: https://scholar.google.es/citations?user=yNjYUycAAAAJ\&hl=es 
Colás-Bravo, P.; Rossi, P. G.; de Pablos-Pons, J.; Conde-Jiménez, J. y Villaciervos Moreno, P. Aplicaciones digitales para la inclusión. El Proyecto Europeo DEPIT

ResearchGate: https://www.researchgate.net/profile/Jesus Conde-Jimenez Academia.edu:

https://independent.academia.edu/Jes\%C3\%BAsCondeJim\%C3\%A9nez

ResearcherID: http://www.researcherid.com/rid/F-6299-2013

Publons: https://publons.com/researcher/2640797/jesus-conde-jimenez/

\section{Patricia Villaciervos Moreno}

Doctora en Ciencias de la Educación y licenciada en Pedagogía. Profesora de la Universidad de Sevilla y de la Universidad Pablo de Olavide (España). Miembro del Grupo de Investigación, Evaluación y Tecnología Educativa (GIETE) HUM-154, desde el año 2004. Integrante de la Red Universitaria de Investigación e Innovación Educativa (REUNI+D). Editora Asociada de la Revista Fuentes y Revisora de otras revistas de investigación de impacto. Investigadora en Proyectos nacionales y europeos. Coordinadora y colaboradora en Proyectos de Innovación y Mejora Docente. Autora de múltiples publicaciones, en libros, capítulos de libros y revistas científicas. Sus líneas de investigación versan sobre aplicaciones de las tecnologías al ámbito educativo; los estereotipos de género; o la inclusión y la calidad educativa.

pvillaciervo@us.es

Orcid ID: https://orcid.org/0000-0001-9812-5060

Google Scholar: https://scholar.google.es/citations?user=tr1tYXUAAAAJ\&hl=es

ResearchGate: https://www.researchgate.net/profile/Patricia Moreno6

Academia.edu: https://us.academia.edu/PatriciaVillaciervosMoreno

ResearcherID: http://www.researcherid.com/rid/K-3867-2017

Publons: https://publons.com/researcher/1635243/p-villaciervos-moreno/ 